\section{Autonomes Fahren - Mobilität und Auto in der Welt von morgen}

\author{
Ausblick zur Akzeptanz des autonomen \\ Fahrens im Projekt „Villa Ladenburg“ \\ der Daimler und Benz Stiftung \\ von Eva Fraedrich, HU Berlin, und Barbara \\ Lenz, DLR
}

Google lässt den Driverless Car medienwirksam über die Straßen von Kalifornien fahren; Daimler beweist mit der Berta-Benz-Fahrt, dass es möglich ist, sich vollautomatisch auf öffentlichen Straßen in Deutschland zu bewegen. Der fahrerlose und gleichzeitig elektrisch angetriebene Nissan Leaf wird auf einer Autobahn in Japan getestet. Man könnte meinen, der Eintritt ins Zeitalter des fahrerlosen Fahrens stünde unmittelbar bevor. Tatsächlich gibt es derzeit aber noch eine große Bandbreite an Fragen, die dringend einer Klärung bedürfen, bevor autonome Straßenfahrzeuge ein fester Bestandteil des Verkehrssystems werden könnten. Neben technischen Herausforderungen wird inzwischen vor allem die rechtliche Problematik der Automation diskutiert (z. B. bei Gasser et al. 2012; Beiker et al. 2012). Gleichzeitig besteht Einigkeit darüber, dass das Thema frühzeitig auch aus dem Blickwinkel der individuellen und gesellschaftlichen Akzeptanz betrachtet werden muss.

\section{Autonomes Fahren - ein Thema mit vielen Facetten}

Arbeiten, die sich mit der Akzeptanz des autonomen Fahrens auseinandersetzen, gibt es bislang erst wenige. Im Wesentlichen handelt es sich dabei um Umfragen zu individuellen Einstellungen gegenüber dem autonomen Fahren. Vereinzelte Arbeiten in den Geistes- und Kulturwissenschaften betrachten die Einbettung der Idee vom fahrerlosen Auto und der , automatisierten Straße“ in die wirtschaftliche und gesellschaftliche Entwicklung von Nationen, insbesondere in den USA (vgl. z. B. Kröger 2012, sowie in diesem Heft). Um ein Bild vom autonomen Fahren zu entwerfen, das über die ausschließliche Berücksichtigung von technischen Aspekten hinausgeht, hat die Daimler und Benz Stiftung das Projekt ,Villa Ladenburg“ ins Leben gerufen. In diesem Projekt arbeitet ein interdisziplinäres Kernteam zu zentralen Fragen, Herausforderungen und Rahmenbedingungen des autonomen Fahrens und ergänzt diese Expertise durch die Zusammenarbeit mit externen Expertinnen und Experten, die ihr themenspezifisches Wissen in fünf Diskursbereiche einbringen (Abb. 1). Damit greift das Projekt die Vielfalt der Fragestellungen und Herausforderungen in einem integrativen Ansatz auf. Die Definition des Begriffes „,autonomes Fahren“ lehnt sich dabei grundsätzlich an die Definition der Bundesanstalt für Straßenverkehrswesen (BASt) zum vollautomatisierten Fahren an (Gasser et al. 2012, S. 9), wonach „vollautomatisiert" bedeutet, dass das System sowohl die Längs- als auch Querführung vollständig übernimmt und die Fahrzeuginsassen von der Aufgabe, das Fahren zu überwachen, entbunden sind. Zur Übertragbarkeit der allgemeinen Definition in realistische Umgebungen erfolgte eine Spezifizierung anhand von konkreten Anwendungsfällen („Use Cases“) (Wachenfeld/Winner 2013), die verschiedene Szenarien aufzeigen, in denen autonom gefahren werden könnte: Autobahnpilot, Autonomes Valet-Parking, Vollautomat mit Verfügbarkeitsfahrer und Vehicle on Demand.

Abb. 1: Diskursbereiche des Projektes, ,Villa Ladenburg" zum Thema Autonomes Fahren

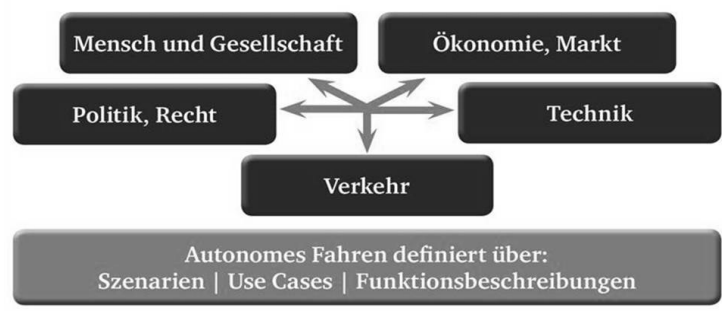

Quelle: Maurer 2013

Im Mittelpunkt des Diskursbereiches „Mensch und Gesellschaft" stehen Fragen der individuellen und gesellschaftlichen Akzeptanz des autonomen Fahrens. Dabei steht die Untersuchung von Akzeptanz allerdings vor dem Problem, dass es bisher gar kein allgemeines Verständnis zum Untersuchungsgegenstand, dem autonomen Fahren, und seinen Eigenschaften gibt - bislang kann autonomes Fahren nur von wenigen ausgewählten Ingenieuren und Entwicklern als Realität wahrgenommen werden. Während bei Untersuchungen, die z. B. das Auto 
zum Gegenstand haben, vergleichsweise ähnliche Vorstellungen bei den Befragten vorliegen, existiert eine solche „kollektive Vorstellungsbasis“ für das autonome Fahrzeug (noch) gar nicht. Aus diesem Grund wurde im Diskursbereich „Mensch und Gesellschaft" in einem ersten Schritt die Frage aufbereitet, welche Aspekte des autonomen Fahrens öffentlich und damit in der Gesellschaft insgesamt sowie individuell derzeit überhaupt wahrgenommen werden (Fraedrich/Lenz 2014; Fraedrich/ Jacobsen 2013). Dazu wurden die Kommentare von Leserinnen und Lesern zu Onlineartikeln von großen deutschen Tageszeitungen und Magazinen analysiert, in denen das autonome Fahren thematisiert wurde. In der explorativ angelegten Untersuchung wurden die Kommentare zu den Artikeln mittels qualitativer Inhaltsanalyse (vgl. Mayring 2010) extrahiert und systematisch ausgewertet. Auf diese Weise konnte ein induktives Kategoriensystem generiert werden, das einen ersten Einblick dazu vermittelt, wie sich autonomes Fahren aus Sicht von Nutzerinnen und Nutzern des Verkehrssystems darstellt. Grundsätzlich verbindet sich mit dieser Herangehensweise auch die Möglichkeit, unterschiedliche Imaginationen von autonomem Fahren zu erfassen, die sich zum Beispiel auch von der oben vorgestellten Definition der BASt unterscheiden; dem wird im Folgenden aber nicht weiter nachgegangen. Vielmehr gibt der Beitrag einen Einblick in die Wahrnehmung der Vielfalt an Aspekten des autonomen Fahrens und stellt sie in Beziehung zu der Frage, welche Hinweise sich daraus zur Akzeptanz von autonomem Fahren ableiten lassen.

\section{Akzeptanz - ein schillernder Begriff}

„Akzeptanz“ ist durch die gleichzeitige Verwendung sowohl im fachwissenschaftlichen $\mathrm{Zu}-$ sammenhang als auch in der Alltagssprache ein äußerst schillernder Begriff, der heutzutage fast inflationär gebraucht wird. Während in der Alltagssprache unter „Akzeptanz" eine mehr oder minder offenkundige Zustimmung zu Personen, Regelungen, Produkten oder Verfahren verstanden wird, gibt es in der Wissenschaft unterschiedliche Theorien und Modelle zum Verständnis von Akzeptanz, die häufig auch auf deren prozesshaften Charakter verweisen. Die meisten Ansätze differenzieren nach unterschiedlichen Dimensi- onen bzw. Ebenen von Akzeptanz: Der Einstellungs-, der Handlungs- bzw. Verhaltens- und der Wertdimension; die Wertedimension wird gelegentlich auch als Teil der Einstellungsdimension begriffen (Schäfer/Keppler 2013).

Arbeiten zur Technikakzeptanz beziehen sich entweder auf gesellschaftliche oder auf individuelle Aspekte des Akzeptanzgegenstands. Auf der individuellen Ebene ist der Ausgangspunkt dann zum Beispiel die Frage nach der Bewertung einer Technologie oder eines konkreten technischen Produktes und gegebenenfalls deren Verbindung mit Nutzungsabsicht und tatsächlicher Nutzung. Neuere Ansätze berücksichtigen auch den Rahmen, in dem einer Technologie erst eine bestimmte Bedeutung zugeschrieben wird und den lebensweltlichen Kontext, in den eine Technologieanwendung eingebettet ist (Lucke 1995; Petermann/Scherz 2005). Was bedeutet das nun hinsichtlich des autonomen Fahrens? Wie oben bereits ausgeführt, ist das autonome Fahren den allermeisten Menschen bisher lediglich durch die mediale Vermittlung ein Begriff. Daher kann die Analyse von Äußerungen zum Thema auch keine Nutzung, bzw. keine Handlungsdimension erfassen. Eine solche explorative Analyse kann aber das Themenfeld strukturieren und dabei - ohne mit den Vorgaben und begrifflichen Vorstrukturierungen eines Fragebogens zu arbeiten - erstens diejenigen Aspekte erfassen, die aus Sicht von potenziellen Nutzerinnen und Nutzer Bedeutung haben und zweitens Einstellungen und Bewertungen ermitteln, die mit der Wahrnehmung des Themas einhergehen.

Im Folgenden wird zunächst ein kurzer Blick auf Befragungsergebnisse geworfen, die in jüngerer Zeit zur Akzeptanz autonomen Fahrens publiziert worden sind, um dann anhand der Analyse von Kommentaren in Onlineforen ein etwas weiteres Bild zum Thema Wahrnehmung von und Einstellungen zum autonomen Fahren zu entwerfen.

\section{Umfragen zu Einstellungen und Nutzungsabsichten}

Die Studien zur Akzeptanz von autonomem Fahren konzentrieren sich bislang auf Einstellungen des individuellen Nutzers, meist verbunden mit der Frage nach einer konkreten (zukünftigen) Nutzungsabsicht. Nachdem die ersten Veröffentlichungen noch ein eher heterogenes Bild aufgewiesen 
hatten, zeigen sich in jüngeren Umfragen zunehmend positive Reaktionen. So nennt eine Umfrage aus dem Jahr 2012 zu Wünschen von Europäerinnen und Europäern an das zukünftige Auto als Ergebnis, dass etwa zwei Drittel der Befragten dem autonomen Fahren gegenüber eher aufgeschlossen seien (AutoScout24 2012). Eine grundsätzliche, aber auch in vielen spezifischen Situationen positive Einstellung zur möglichen Nutzung eines „Autopiloten“ berichtet auch die Nachfolge-Umfrage des europäischen Automobilportals „Unser Auto von morgen 2013/14“ (AutoScout24 2013). Diese Ergebnisse passen grundsätzlich zur Aussage der Continental Mobilitäts-Studie (2013), in der 53 Prozent der Befragten in Deutschland der Aussage „Automatisiertes Fahren ist ein nützlicher Fortschritt" zustimmen, wenngleich auch eine ganze Reihe von Vorbehalten bestehen. Interessant ist in diesem Zusammenhang auch, dass laut Continental-Studie immerhin 67 Prozent der Befragten mit dem, in der Studie verwendeten Begriff, ,automatisiertes Fahren" etwas anfangen können.

Der Nutzungskontext des autonomen Fahrens wird abseits von gängigen Vorstellungen zum Autofahren bisher noch kaum ausreichend berücksichtigt und geht eigentlich nicht über direkt mit dem autonomen Fahren assoziierte Eigenschaften hinaus. Das heißt, der wirkliche „Akzeptanzkontext", der für unterschiedliche Personengruppen unterschiedlich ausgeprägt ist, wird nicht gewürdigt. So kommt es in einzelnen Studien auch zu Beobachtungen, die zunächst überraschen. In der Deloitte-Studie von 2011 beispielsweise geben jüngere Fahrerinnen und Fahrer zwischen 19 und 31 Jahren an, dass sie das Fahren selbst oft als lästig empfinden. Denn die Fahraufgabe verhindert, dass sie sich während der Fahrt Beschäftigungen zuwenden können, die ihnen eigentlich als wichtiger erscheinen - wie z. B. das Schreiben von Textnachrichten:

„Regulation keeps trying to say texting is distracting to driving but for the consumer it is really the driving that is distracting to texting." (Deloitte 2011, S. 2)

\section{Autonomes Fahren: Welche Aspekte finden Beachtung?}

Die Analyse der Reaktion von Leserinnen und Lesern auf Artikel mit dem Thema „Autonomes Fah- ren" in den Onlineausgaben großer nationaler Zeitungen und Zeitschriften liefert die Grundlage, um das Themenfeld „Autonomes Fahren“ nach den derzeit wahrgenommenen Aspekten zu strukturieren. Eine solche Herangehensweise berücksichtigt die Annahme, dass der mediale Diskurs einen entscheidenden Einfluss auf die individuelle und gesellschaftliche Meinungsbildung hat (vgl. Jäger/ Jäger 2007, S. 23; Dreesen et al. 2012, S. 11ff.). Deshalb wurden für die Analyse Nachrichtenportale ausgewählt, die solchen Tageszeitungen und Magazinen zuzuordnen sind, deren Printausgaben mindestens überregional erscheinen und damit ein repräsentatives Bild der deutschen Medienlandschaft widerspiegeln - gleichzeitig kann so auch davon ausgegangen werden, dass diese Portale in der Lage sind, den aktuellen Diskurs zum autonomen Fahren (mit) zu prägen. Bei den Portalen handelt es sich um BILD.de, Die Welt, Frankfurter Allgemeine Zeitung, Spiegel Online, Sueddeutsche.de und Zeit Online. Um eine möglichst große Ähnlichkeit der Artikel zu gewährleisten, auf die sich die Kommentare bezogen, wurden in einem Querschnitt nur Artikel, welche die Straßenzulassung von autonomen Fahrzeugen in Kalifornien Ende September 2012 thematisierten, herangezogen - ein Ereignis, das medial große Resonanz erfuhr. Über die kommentierenden Leserinnen und Leser und deren soziodemografischen Hintergrund können aufgrund der Anonymität beim Verfassen von Onlinekommentaren - üblicherweise durch die Angabe von Fantasienamen - grundsätzlich wenig bis keine Angaben gemacht werden. Dieses Fehlen von „Rahmendaten“ ist im Zusammenhang mit der Analyse von Onlinedaten durchaus problematisch. Gleichzeitig hat sich das Internet mit seiner schier unbegrenzten und leicht zugänglichen Fülle an Daten auch für qualitative Auswertungsverfahren in den letzten Jahren zu einem wachsenden Forschungsfeld entwickelt (Dresing/Kuckartz 2007, S. 144ff.). Um Muster und Strukturen in den Onlinekommentaren vor dem Hintergrund der Anonymität trotzdem größtmöglich $\mathrm{zu}$ validieren, wurde ein theoretisches Sampling durchgeführt (Lamnek 2010, S. 193ff.). Dazu wurden drei Vergleichsgruppen gebildet - Kommentar auf deutschen Massenmedienportalen, Kommentare auf US-amerikanischen Massenmedienportalen und Kommentare auf einem 
(deutschen) technologiespezifischen bzw. -affinen Portal. Im Folgenden werden wir uns nur auf die Analyse der Kommentare auf deutschen Massenmedienportalen beziehen (s. o.). Die Auswertung der Kommentare erfolgte mittels der qualitativen Inhaltsanalyse nach Mayring (2010), einem etablierten Standardverfahren innerhalb der qualitativen Sozialforschung, dessen Ziel es ist, die Bedeutungen von Texten systematisch und intersubjektiv überprüfbar zu erschließen. Insgesamt wurden 314 Kommentare ausgewertet und mit 538 sog. „Codings“, das heißt Textpassagen mit spezifischem Inhalt, versehen. Die 314 Kommentare lassen sich wiederum 214 ,Fällen“ zuordnen, also kommentierenden Leserinnen und Lesern. Ergebnis der Analyse ist ein Kategoriensystem, das sich auf zwei Ebenen verteilt:

Auf einer sachlich-objektbezogenen Ebene finden sich wahrgenommene oder erwartete Eigenschaften von autonomem Fahren bzw. autonomen Fahrzeugen; dabei orientieren sich die Kommentare eher an funktional-rationalen Überlegungen zur Autonutzung der Zukunft. Aussagen dieser Ebene beziehen sich auf das Objekt, also das autonome Fahrzeug oder das autonome Fahren. Die Hauptkategorien auf dieser Ebene sind wahrgenommene Eigenschaften autonomer Fahrzeuge und autonomen Fahrens (in der Differenzierung positiv, negativ), Überlegungen zu Haftung, Versicherung und Recht sowie Entwicklungsperspektiven von Autonutzung und Autobesitz.

Die zweite Ebene umfasst affektiv-subjektbezogene Aussagen, die sich auf gefühlsmäßige Einstellungen und Bewertungen gegenüber dem autonomen Fahren bzw. gegenüber der Autonutzung und dem Autobesitz allgemein beziehen. In der Regel wird in diesen Äußerungen ein direkter Bezug zur eigenen Person bzw. der eigenen Autonutzung - im Heute oder Morgen - hergestellt. Die Hauptkategorien auf dieser Ebene sind bewertende Einstellungen und Erwartungen zum autonomen Fahren (in der Differenzierung negativ, positiv, ambivalent) sowie die Motivation für Autonutzung und Autobesitz.

Gegenstand der nachfolgenden Ausführungen sind die Hauptkategorien „Eigenschaften autonomer Fahrzeuge und des autonomen Fahrens" und „Bewertende Einstellungen und Erwartungen“.

\section{Wie wird das autonome Auto wohl sein?}

Insgesamt zeigt die Analyse der in den Foren geposteten Kommentare eine durchaus differenzierte Auseinandersetzung mit dem Thema „Autonomes Fahren“ und „Autonome Fahrzeuge“. Rund ein Viertel aller Kommentare bezieht sich auf die Eigenschaften autonomer Fahrzeuge und die Auswirkungen des autonomen Fahrens auf das Verkehrssystem. Die Foristinnen und Foristen äußern dezidierte Erwartungen hinsichtlich der Eigenschaften der Fahrzeuge, aber auch hinsichtlich der möglichen Veränderungen des Verkehrssystems (Tab. 1). Dabei zeigt sich, dass positive Erwartungen deutlich überwiegen - 70 Prozent der sachlich-objektbezogenen Äußerungen lassen sich als positiv einordnen.

Tab. 1: Erwartete Eigenschaften autonomer Fahrzeuge und Auswirkungen des autonomen Fahrens auf das Verkehrssystem $(\mathrm{N}=137)$

\begin{tabular}{|c|c|c|}
\hline $\begin{array}{l}\text { Eigenschaften autonomer } \\
\text { Fahrzeuge } \\
\text { Kategorie „, sachlich/ob- } \\
\text { jektbezogen" }\end{array}$ & $\begin{array}{c}\text { Nennungen } \\
\text { absolut }\end{array}$ & $\begin{array}{l}\text { Nennung in } \\
\text { Prozent }\end{array}$ \\
\hline positiv & 97 & 71 \\
\hline $\begin{array}{l}\text { Sicherheit \& Systemver- } \\
\text { lässlichkeit }\end{array}$ & 38 & 39 \\
\hline Flexibilität \& Komfort & 27 & 28 \\
\hline Verkehrsoptimierung & 10 & 10 \\
\hline $\begin{array}{l}\text { Integrative Verkehrsteil- } \\
\text { nahme }\end{array}$ & 8 & 8 \\
\hline Fortschritt & 5 & 5 \\
\hline Nachhaltigkeit & 5 & 5 \\
\hline Kostenersparnis & 4 & 4 \\
\hline negativ & 40 & 29 \\
\hline $\begin{array}{l}\text { gesellschaftliche Konse- } \\
\text { quenzen }\end{array}$ & 19 & 48 \\
\hline Datenmissbrauch & 7 & 18 \\
\hline $\begin{array}{l}\text { Mängel technischer Infra- } \\
\text { struktur }\end{array}$ & 6 & 15 \\
\hline Teuerung & 4 & 10 \\
\hline Unklarheiten & 4 & 10 \\
\hline Summe der Nennungen & 137 & 100 \\
\hline
\end{tabular}

Quelle: verändert nach Fraedrich/Lenz 2014

Den autonomen Fahrzeugen werden in den Foren-Kommentaren Systemeigenschaften zugeschrieben, die entweder eher positive oder eher negative Aspekte beschreiben. Im Bereich der positiven Zuschreibungen rangieren die Kategorien „Sicherheit \& Systemverlässlichkeit“" sowie „Fle- 
xibilität \& Komfort" an erster Stelle. Im Zusammenhang mit Sicherheit und Systemverlässlichkeit wird vor allem auf den Vorteil abgehoben, dass computergestützte Systeme im Vergleich mit menschlichen Fahrern weniger Fehler machen dass durch die Automation also die zahlreichen Verkehrsunfälle, die auf menschliches Versagen bzw. Fehlverhalten zurückzuführen sind, vermieden werden könnten. Die Foristinnen und Foristen nehmen an, dass autonome Fahrzeuge mit ihren, den menschlichen Wahrnehmungs- und Reaktionsfähigkeiten weit überlegenen Eigenschaften in Zukunft helfen können, Unfälle im Straßenverkehr gänzlich zu verhindern oder sie zumindest stark zu reduzieren. Der besondere Nutzen des autonomen Fahrens wäre damit das sichere Fahren.

Unter „Flexibilität \& Komfort“ wird verstanden, dass autonomes Autofahren unter Bedingungen möglich ist, die "normales" Fahren vorher nicht zugelassen haben. Flexibel sein bedeutet in dieser Hinsicht, sich künftig mit anderen Dingen beschäftigen zu können, als das heute beim Autofahren der Fall ist. Als „Fahrerin“ oder „Fahrer“ muss man nicht mehr auf den Verkehr achten und das Fahrzeug bedienen. Diese Art der Flexibilität macht Autofahren gleichermaßen komfortabler und bequemer. Gleichzeitig werden Ressourcen für andere Tätigkeiten frei. Nutzerinnen und Nutzer autonomer Fahrzeuge könnten im Auto arbeiten, lesen, Filme ansehen oder auch mehr freie Zeit haben, unter anderem weil das Auto schneller am Zielort ankommt als im gegenwärtigen RushHour-Verkehr. Eine solche „Befreiung“ vom oftmals als lästig wahrgenommenen Fahren wird als (gesellschaftlicher) Fortschritt artikuliert, der mit der neuen Technologie einhergeht. Darüber hinaus erwarten die Foristinnen und Foristen, dass auch Personen, die unter den gegenwärtigen Bedingungen gar nicht oder nur eingeschränkt ein Auto nutzen können, mit Hilfe des autonomen Fahrens in Zukunft mit einem Individual-Fahrzeug mobil sein können, also zum Beispiel alte, blinde oder anderweitig eingeschränkte Menschen. Unter ,anderweitig eingeschränkten Personen" werden hier auch jene verstanden, die nicht mehr selbst fahren können oder dürfen, weil sie zu einem bestimmten Zeitpunkt unter Alkohol-, Drogen- oder Medikamenteneinfluss stehen.
Kostenersparnisse sind eine weitere positive Eigenschaft, die in den Foren thematisiert wird. Damit werden einerseits Möglichkeiten bezeichnet, durch die flexible und bequeme Nutzung eines autonomen Fahrzeugs nicht mehr für andere Verkehrsmittel zahlen zu müssen (z. B. weil nach einer langen Disconacht kein teures Taxi mehr in Anspruch genommen werden muss), sowie andererseits die Reduktion von Spritkosten durch eine vorausschauende computergesteuerte Fahrweise. Letzteres wird häufig auch im Zusammenhang mit dem Thema „Nachhaltigkeit" geäußert.

Überhaupt ist die Diskussion in den Foren keineswegs nur vom individuellen Nutzen gekennzeichnet, den sich die Schreiberinnen und Schreiber der Kommentare erwarten. Vielmehr gehen die Überlegungen durchaus auch in Richtung der Möglichkeiten und Chancen, die sich für das Verkehrssystem insgesamt aus dem Einsatz autonomer Fahrzeuge ergeben. So wird die Erwartung ausgesprochen, dass der Verkehr der Zukunft dank der autonomen Fahrzeuge entlastet und verflüssigt wird, v. a. weil Staus maßgeblich reduziert oder sogar komplett eliminiert werden, z. B. dadurch, dass Abstände zwischen den einzelnen Autos generell verringert werden. Damit verbindet sich oft die Vorstellung, dass „Computersysteme“ zentral gesteuert und damit Baustellen, Witterungsbedingungen, dichte Verkehrszeiten etc. in die Routenführung eingeplant und Fahrzeuge automatisch geleitet werden könnten. Darüber hinaus wird angenommen, dass autonome Fahrzeuge spritsparend und damit emissionsarm sind und somit dazu beitragen, verkehrsbedingte Umweltbelastungen zu reduzieren. Schließlich erwarten die Foristinnen und Foristen, dass durch autonome Systeme auch das Fahrzeugdesign revolutioniert werden könnte - hin zu leichteren oder gar kleineren Fahrzeugen, nicht nur, weil der Innenraum eines Autos in Zukunft nicht mehr den gleichen Anforderungen entsprechen müsste wie in einem konventionellen Fahrzeug, der für Fahrerin oder Fahrer ein Cockpit benötigt, sondern auch, weil auf schwere und platzraubende Sicherheitseinbauten verzichtet werden könnte. Auch davon versprechen sich viele einen nachhaltigeren Verkehr, der Umwelt und Klima künftig weniger stark belastet. In den Abbildungen 2 und 3 wurden beispielhaft solche Vorstellungen vom autonomen Fahrzeug zeichnerisch umgesetzt. 
Hier wird auch deutlich, dass die Wahrnehmungen darüber, was ein autonomes Fahrzeug eigentlich ist, teilweise deutlich divergieren - während einige sich unter autonomem Fahren eher ein Auto im „konventionellen“ Sinn vorstellen, bei dem auf Wunsch die Fahraufgabe an das Fahrzeug abgegeben werden kann, imaginieren andere wiederum eine Form der Fortbewegung, die unser heutiges Verständnis von Autonutzung und -besitz deutlich revolutionieren würde.

\section{Abb. 2: Vehicle on Demand}

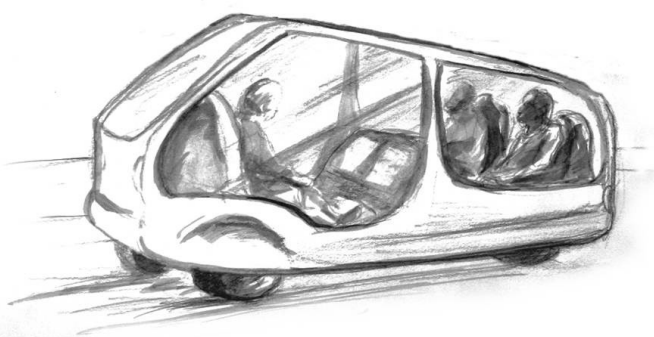

Zeichnung: Lenz 2013

Wenngleich die Wahrnehmung von positiven Eigenschaften autonomen Fahrens bzw. autonomer Fahrzeuge deutlich überwiegt, gibt es auch eine Reihe von Kommentaren, in denen Szenarien entwickelt werden, die auf erwarteten negativen Eigenschaften der autonomen Autos der Zukunft aufbauen. Dabei stehen weniger persönliche Nachteile im Vordergrund als vielmehr gesellschaftliche Konsequenzen des autonomen Fahrens. Befürchtungen, die geäußert werden, betreffen das Problem von Arbeitsplatzverlusten in Dienstleistungsbranchen, der Transportlogistik oder in der Automobilherstellung, die Angst vor einer zu starken Einflussnahme von Wirtschaftsunternehmen auf politische Entscheidungsprozesse, sowie die Nähe von militärischen Institutionen zur Alltagsmobilität. Auch die Sorge vor einer zunehmenden Technisierung der menschlichen Lebenswelt wird als mögliche negative Eigenschaft autonomen Fahrens in den Blick genommen. Gesondert diskutiert wird eine möglicherweise drohende intensive Überwachung des Einzelnen angesichts der hohen Abhängigkeit des Systems von Daten zur Erfassung und Steuerung der Fahrzeuge. Damit wird auch Datenmissbrauch und sogar die Möglichkeit eines Angriffs auf das hochsensible und potenziell risikobehaftete System durch Hacker bzw. Terroristen in Verbindung gebracht.

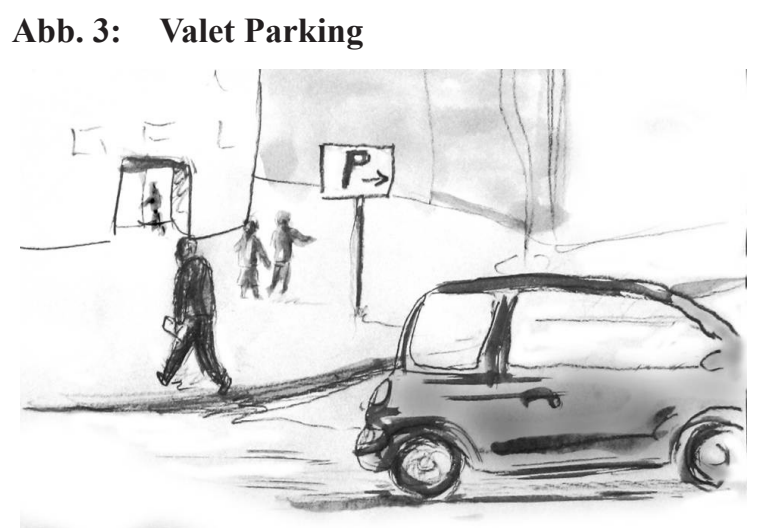

Zeichnung: Lenz 2013

Unter der Überschrift Mängel technischer Infrastruktur sind Befürchtungen zusammengefasst, dass ein autonomes Fahrzeug, wie andere Computersysteme auch, einen Systemabsturz erleiden könnte - mit möglicherweise fatalen Konsequenzen. Vermutet wird, dass menschliche Fahrerinnen und Fahrer viel besser in der Lage sind, eventuellen Gefahren- und Unfallquellen im Straßenverkehr zu begegnen als ein Computer. Die Foristinnen und Foristen äußern die Ansicht, dass schließlich auch ein als sicher deklariertes System wie das autonome Fahrzeug auf der Leistung menschlicher Softwareentwickler und Programmierer basiert, die nicht immer fehlerfrei arbeiten und somit die Sicherheit im Straßenverkehr gefährden könnten.

Eine weitere negative Annahme, die auf das autonome Fahrzeug projiziert wird, ist die Sorge vor einer Verteuerung des Autofahrens entweder, weil die aufwendige Computertechnik das Auto der Zukunft unerschwinglich machen könnte oder aber, weil die Versicherungsbeiträge evtl. höher sein könnten als bei herkömmlichen Fahrzeugen. Damit impliziert ist die Annahme, dass Versicherungen dem Menschen mehr trauen werden als der vollautomatisierten Maschine. Als Folge erhöhter Versicherungsbeiträge wird befürchtet, dass nur ein kleiner Kreis besonders wohlhabender Menschen sich autonomes Fahren überhaupt leisten können wird.

Ergänzt man die eher sachbezogenen Äußerungen der Foristinnen und Foristen durch deren wertenden Einstellungen und Erwartungen, dann 
zeigen sich ganz unterschiedliche Gründe für eine ablehnende Haltung. In den eher grundsätzlich kommentierenden Beiträgen zeigt sich, dass die Kontrolle über die Fahrzeugsteuerung ungern abgegeben wird oder dass der Sicherheit und $\mathrm{Zu}$ verlässigkeit des noch neuen, wenig erprobten Systems wenig Vertrauen entgegengebracht wird. Daneben stehen Aussagen, in denen der Realisierung autonomen Fahrens im Straßenverkehr mit Misstrauen und Skepsis entgegengeblickt wird. So werden Zweifel an der Umsetzbarkeit oder Machbarkeit von autonomen Fahrsystemen geäußert. Skepsis existiert auch gegenüber der generellen Sinnhaftigkeit von autonomem Fahren, nicht zuletzt weil bereits Taxi, Bus oder Bahn Vorteile bieten, wie sie autonome Fahrzeuge versprechen. Darüber hinaus werden Zweifel bzgl. der Technikentwicklung genannt. Dabei spielen negative Erfahrungen mit anderen technischen Neuerungen, aber auch mit bisherigen Fahrerassistenzsystemen eine Rolle, denen nachgesagt wird, nicht oder nur unzureichend zu funktionieren und damit sogar gefährliche Situationen verursachen zu können. Einzelne Foristinnen und Foristen sind der Meinung, dass es durchaus Ausnahmesituationen geben könnte, in denen Menschen Maschinen überlegen seien.

Daneben stehen Kommentare, in denen dem autonomen Fahren mit Optimismus und Fortschrittsvertrauen entgegengesehen wird. Diese Äußerungen gründen meist auf der Überzeugung, dass nach einer gewissen Phase der Gewöhnung autonomes Fahren genauso selbstverständlich zur Lebenswelt dazugehören wird, wie andere, ehemals revolutionäre technische Neuerungen, wie beispielsweise das Mobiltelefon, das Internet oder Fahrerassistenzsysteme. Mit dem autonomen Fahren verbindet sich die Hoffnung, dass das Leben einfacher und das Reisen flexibler wird, und dass die Menschen mehr Zeit für sinnvollere Dinge haben, wenn sie die lästige Fahraufgabe los sind. Dem steht die Gruppe derjenigen gegenüber, für die Faktoren wie Fahrspaß und Kontrolle über die Steuerung eines Fahrzeugs eine wesentliche Motivation für die Autonutzung sind. Insgesamt sind die Einstellungen eher negativ: Rund 42 Prozent der wertenden Äußerungen sind eindeutig negativ, wobei v. a. die technologische Realisierbarkeit in Zweifel gezogen wird. Zehn Prozent der werten- den Kommentare bringen eine eindeutige Ablehnung des autonomen Fahrens zum Ausdruck.

\section{Fazit}

Ganz offensichtlich hat eine breite Diskussion um das autonome Autofahren begonnen - breit zumindest im Hinblick auf die Themen, die dabei angesprochen werden. Die Analyse der Onlineforen hat gezeigt, dass - über ganz unterschiedliche Plattformen hinweg - die Diskussion nicht nur die Frage nach dem individuellen Nutzen stellt, die in der Akzeptanzforschung meist besonders hervorgehoben wird. Vielmehr gehen auch Aspekte, die den Verkehr und das Verkehrssystem insgesamt betreffen, in die Kommentare und Diskussionen ein. Dies lässt eine spannende Auseinandersetzung mit dem Thema „Autonomes Fahren" auch für die Zukunft erwarten.

Gleichzeitigt zeigt aber auch die rege Debatte um Rahmenbedingungen des autonomen Fahrens, dass es im Zusammenhang mit dem Auto der Zukunft nicht nur darum geht, das Automobil, wie wir es heute kennen, technisch weiterzuentwickeln und sicherer, komfortabler, besser und auch umweltschonender zu machen. Auf der Diskursagenda steht vielmehr auch die Frage, was das autonome Fahren an gesellschaftlichen Auswirkungen mit sich bringen würde und damit verbunden, wie wir uns in der Welt von morgen bewegen wollen.

\section{Literatur}

AutoScout24 GmbH, 2012: Unser Auto von morgen. Studie zu den Wünschen der Europäer an das Auto von morgen; http://about.autoscout24.com/de-de/ au-press/2012_as24_studie_auto_v_morgen_en.pdf (download 9.7.13)

AutoScout24 GmbH, 2013: Unser Auto von morgen 2013/14; http://ww2.autoscout24.de/special/unserauto-von-morgen--2013-14/was-wuenschen-sichdie-europaeer-vom-auto-von-morgen/4319/392974/ (download 14.4.14)

Beiker, S., 2012: Legal Aspects of Autonomous Driving. In: Chen, L.K.; Quigley, S.K.; Felton, P.L.; Roberts, C.; Laidlaw, P. (Hg.): Driving the Future: The Legal Implications of Autonomous Vehicles. In: Santa Clara Review 52/4, S. 1145-1156; http://digitalcommons.law. scu.edu/lawreview/vol52/iss4/ (download 23.4.14)

Continental Mobilitäts-Studie, 2013: http://www. continental-corporation.com/www/download/presseportal_com_de/allgemein/automatisiertes_fahren_de/ 
ov_mobilitaetsstudie_2013/download_channel/ praes_mobilitaetsstudie_de.pdf (download 14.4.14)

Deloitte, 2011: Gaining speed: Gen Y in the Driver's Seat. Third Annual Deloitte Automotive Generation Y Survey

Dreesen, P.; Kumięga, L.; Spieß, C. (Hg.), 2012: Mediendiskursanalyse. Diskurse - Dispositive - Medien - Macht. Wiesbaden

Dresing, Th.; Kuckartz, U., 2007: Neue Datenquellen für die Sozialforschung: Analyse von Internetdaten. In: Kuckartz, U.; Grunenberg, H.; Dresing, Th. (Hg.): Qualitative Datenanalyse: computergestützt, S. 143-162

Fraedrich, E.; Jacobsen, M., 2013: Autonomous Driving - Challenges in Research Concerning Future Acceptance. Presentation at European Transport Conference 2013, Frankfurt a. M.

Fraedrich, E.; Lenz, B., 2014: Automated Driving Individual and Societal Aspects Entering the Debate. Paper presented at TRB 93rd Annual Meeting

Gasser, T.; Arzt, C.; Ayoubi, M. et al., 2012: Rechtsfolgen zunehmender Fahrzeugautomatisierung. Gemeinsamer Schlussbericht der Projektgruppe. In: Berichte der Bundesanstalt für Straßenwesen. Fahrzeugtechnik F 83. Bremerhaven

Hüsing, B.; Bierhals, R.; Bührlen, B. et al., 2002: Technikakzeptanz und Nachfragemuster als Standortvorteil. Abschlussbericht an das Bundesministerium für Bildung und Forschung. Karlsruhe

Jäger, M.; Jäger, S., 2007: Deutungskämpfe. Theorie und Praxis Kritischer Diskursanalyse. Wiesbaden

Kröger, F., 2012: Fahrerlos und unfallfrei. Eine frühe automobile Technikutopie und ihre populärkulturelle Bildgeschichte. In: Fraunholz, U.; Woschech, A. (Hg.): Technology Fiction. Technische Visionen und Utopien in der Hochmoderne. Bielefeld, S. 93-114

Lamnek, S., 2010: Qualitative Sozialforschung. Weinheim

Lenz, J., 2013: Zeichnung. In: Fraedrich, E.; Hoor, M.; Jacobsen, M.: Autonomes Fahren. Ein Zukunftsszenario zur Diskussionsrunde. Humboldt-Universität zu Berlin

Lucke, D., 1995: Akzeptanz. Legitimität in der Abstimmungsgesellschaft. Opladen

Maurer, M., 2013: Autonome Automobile - Wer steuert das Fahrzeug der Zukunft? Dialog im Museum. Vortrag. Stuttgart, S. 36; https://www.daimlerbenz-stiftung.de/cms/forschung/foerderprojekte.html (download 23.4.14)

Mayring, P., 2010: Qualitative Inhaltsanalyse. Grundlagen und Techniken. Weinheim
Petermann, Th.; Scherz, C., 2005: TA und (Technik-) Akzeptanz(-forschung). In: Technikfolgenabschätzung - Theorie und Praxis 14/3 (2005), S.45-53

Schäfer, M.; Keppler, D., 2013: Modelle der technikorientierten Akzeptanzforschung. Überblick und Reflexion am Beispiel eines Forschungsprojekts zur Implementierung innovativer technischer Energieeffizienz-Maßnahmen. Discussion Paper Nr. 34/2013, Zentrum Technik und Gesellschaft, Technische Universität Berlin

Wachenfeld, W.; Winner, H., 2013: Use Cases des Autonomen Fahrens. Release1 vom 24.12.2013; https:// www.daimler-benz-stiftung.de/cms/forschung/foerderprojekte.html (download 23.4.14)

\section{Liste der ausgewählten Portale}

BILD.de, 2012: Freie Fahrt für Googles Roboter-Autos, 26.9.12; http://www.bild.de/digital/multimedia/ google/google-auto-darf-fahren-26404736.bild.html (download 22.4.14)

Die Welt - Doll, N., 2012: Selbstlenkendes Auto kommt schneller als man denkt, 26.9.12; http:// www.welt.de/wirtschaft/article109473825/Selbstlenkendes-Auto-kommt-schneller-als-man-denkt. html\#disqus_thread (download 22.4.14)

Frankfurter Allgemeine Zeitung, 2012: Kalifornien lässt fahrerlose Autos im Straßenverkehr zu, 26.9.12; http://www.faz.net/aktuell/technik-motor/google-auto-kalifornien-laesst-fahrerlose-autos-im-strassenverkehr-zu-11904259.html (download 22.4.14)

Spiegel Online - Büttner, R., 2012: Neues Gesetz in den USA: Kalifornien lässt autonome Pkw auf die Straßen, 26.9.12; http://www.spiegel.de/auto/aktuell/ neues-gesetz-in-kalifornien-duerfen-autonome-autoauf-die-strassen-a-857988.html (download 22.4.14)

Süddeutsche.de - Crocoll, S., 2012: Hilf mir, Kumpel, 27.9.12; http://www.sueddeutsche.de/auto/autosdie-sich-selbst-steuern-hilf-mir-kumpel-1.1479826 (download 22.4.14)

Zeit Online - Biermann, K., 2012: Google: Kalifornien lässt autonome Autos auf die Straße, 26.9.12; http://www.zeit.de/digital/mobil/2012-09/google-autonome-autos (download 18.6.13)

\section{Kontakt}

Eva Fraedrich, M.A.

Geographisches Institut

Humboldt-Universität zu Berlin

Unter den Linden 6, 10099 Berlin

Tel: +49 30 2093-6863

E-Mail: eva.fraedrich@geo.hu-berlin.de

$\ll »$ 